\title{
Coincidence Problem and Holographic $f(R)$ Gravity in Spatially flat and Curved Universes
}

\author{
Yousef Bisabr* \\ Department of Physics, Shahid Rajaee Teacher Training University, Lavizan, Tehran 16788, Iran
}

\begin{abstract}
The $f(R)$ gravity models formulated in Einstein conformal frame are equivalent to Einstein gravity together with a minimally coupled scalar field. The scalar field couples with the matter sector and the coupling term is given by the conformal factor. We apply the holographic principle to such an interacting model in spatially flat and curved universes. We show that the model leads to a constant ratio of energy densities of dark matter to dark energy in a spatially flat universe. In a spatially curved universe, the ratio is not a constant and the evolution seems to be model-dependent. However, we argue that any cosmologically viable $f(R)$ model can lead to a nearly constant ratio of energy densities and therefore alleviate the coincidence problem.
\end{abstract}

It is now generally believed that the universe is currently undergoing a period of accelerated expansion. The simplest candidate to produce this cosmic acceleration is the cosmological constant, the energy density associated with quantum vacuum. However, there are several problems for associating cosmic acceleration with the cosmological constant. First, theoretical estimates on its value are many order of magnitude larger than observations [1]. Second, it is simply a constant, namely that it is not diluted with expansion of the universe. This latter is specifically important in the sense that there are observational evidence [2] demonstrating that the cosmic acceleration is a recent phenomena and the universe must have passed through a deceleration phase in the early stages of its evolution. This deceleration phase is important for successful nucleosynthesis as well as for the structure formation. We therefore need a field evolving during expansion of the universe in such a way that its dynamics makes the deceleration parameter have a signature flip from positive in the early stages of matter dominated era to negative in the present stage [3]. There is also another problem which is the focus of the present note. It concerns with the coincidence between the observed vacuum energy density

*e-mail: y-bisabr@srttu.edu. 
and the current matter density. While these two energy components evolve differently as the universe expands, their contributions to total energy density of the universe in the present epoch are the same order of magnitude.

As a different point of view, cosmic acceleration may be interpreted as evidence either for existence of some exotic matter components or for modification of the gravitational theory. In the first route of interpretation one can take a mysterious cosmic fluid with sufficiently large and negative pressure, dubbed dark energy. These models are usually invoked a scalar field which during its evolution takes negative pressure by rolling down a proper potential. In the second route, however, one attributes the accelerating expansion to a modification of general relativity. A particular class of models that has recently drawn a significant amount of attention is the so-called $f(R)$ gravity models [4]. These models propose a modification of Einstein-Hilbert action so that the scalar curvature is replaced by some arbitrary function $f(R)$.

Recently, different models inspired by holographic principle have been proposed to explain the cosmic acceleration. The basic idea is that the number of degrees of freedom of a physical system scales with its bounding area rather than with its volume [5]. For an effective quantum field theory in a box of size $L$ with an ultraviolet (UV) cutoff $\Lambda$, the entropy $S$ scales extensively as $S \sim L^{3} \Lambda^{3}$. However the peculiar thermodynamics of black holes has led Bekenstein [6] to postulate that the maximum entropy in a box of volume $L^{3}$ behaves non-extensively, growing as the area of the box. In this sense there is a so-called Bekenstein entropy bound

$$
S=L^{3} \Lambda^{3} \leq S_{B H} \equiv \pi L^{2} M_{p}^{2}
$$

where $S_{B H}$ is the entropy of a black hole of radius $L$, and $M_{p} \equiv(8 \pi G)^{-\frac{1}{2}}$ stands for the reduced Planck mass. It is important that in this relation the length scale $L$ providing an Infrared (IR) cutoff is determined by the UV cutoff $\Lambda$ and can not be chosen independently. However such a non-extensive scaling law seems to provide a breakdown of quantum field theory at large scales. To reconcile this breakdown with the success of local quantum field theory in describing observed particle phenomenology, Cohen et al. [7] proposed a more restrictive bound. Since the maximal energy density in the effective theory is of the order $\rho_{\Lambda}=\Lambda^{4}$, requiring that the energy in a given volume not to exceed the energy of a black hole of the same size results in the constraint

$$
L^{3} \rho_{\Lambda} \leq L M_{p}^{2}
$$

If we take the largest value of the length scale $L$ as the IR cutoff saturating the inequality (2), we then obtain the holographic dark energy density

$$
\rho_{\Lambda}=3 c^{2} M_{p}^{2} L^{-2}
$$

in which $3 c^{2}$ is a numerical constant. It is interesting to note that if the length scale $L$ is characterized by the size of the universe, the Hubble scale $H^{-1}$, then equation (3) gives a vacuum energy density of the right order of magnitude consistent with observations [7]. It is however pointed out that this yields a wrong equation of state parameter for dark energy, and other possible values for $L$ should be chosen such as the size of the future event horizon [8]. This conclusion is, however, based on the assumption of an independent evolution of energy 
densities of dark energy and dark matter. It is shown [9] that, if there is any interaction between these two components the identification of $L$ with $H^{-1}$ is possible. In particular, the authors of [9] [10] argued that such an identification necessarily implies a constant ratio of the energy densities of the two components regardless of the details of the interaction.

In the present note, we investigate the coincidence problem in the context of holographic $f(R)$ gravity models. In $f(R)$ models the dynamical variable of the vacuum sector is the metric tensor and the corresponding field equations are fourth order. This dynamical variable can be replaced by a new pair which consists of a conformally rescaled metric and a scalar partner. Moreover, in terms of the new set of variables the field equations are those of General Relativity. The original set of variables is commonly called Jordan conformal frame and the transformed set whose dynamics is described by Einstein field equations is called Einstein conformal frame. The dynamical equivalence of Jordan and Einstein conformal frames does not generally imply that they are also physically equivalent. In fact it is shown that some physical systems can be differently interpreted in different conformal frames [11] [12]. The physical status of the two conformal frames is an open question which we are not going to address here.

We will work in Einstein conformal frame. The motivation is that in this frame there is a coupling between the scalar degree of freedom and matter sector induced by the conformal transformation. In this context, we have already studied the coincidence problem without any use of holographic principle [13]. It is shown that the requirement of a constant ratio of energy densities of the two components, puts some constraints on the functional form of the $f(R)$ function. In other terms, there is a class of parameterized $f(R)$ models for which the coincidence problem may be alleviated within a particular region of the parameters space.

Applying the holographic principle to $f(R)$ gravity leads to some important changes in this result. We show that identification of IR cutoff with the Hubble scale in a spatially flat universe necessarily leads to a stationary ratio of energy densities corresponding to dark energy and matter sector regardless of the functional form of the $f(R)$ function. Thus, any holographic $f(R)$ model can address the coincidence problem in a spatially flat universe. In a universe with a spatial curvature, we will show that the ratio of energy densities is no longer a constant and evolves during expansion of the universe. We argue that alleviation of the coincidence problem requires conditions that directly link to the cosmological viability of $f(R)$ models. Let us start with introducing the action for an $f(R)$ gravity theory in the Jordan frame

$$
S_{J F}=\frac{1}{2} \int d^{4} x \sqrt{-g} M_{p}^{2} f(R)+S_{m}\left(g_{\mu \nu}, \psi\right)
$$

where $g$ is the determinant of $g_{\mu \nu}$ and $S_{m}$ is the action of (dark) matter which depends on the metric $g_{\mu \nu}$ and some (dark) matter field $\psi$. Stability in matter sector (the Dolgov-Kawasaki instability [14]) imposes some conditions on the functional form of $f(R)$ models. These conditions require that the first and the second derivatives of $f(R)$ function with respect to the Ricci scalar $R$ should be positive definite. The positivity of the first derivative ensures that the scalar degree of freedom is not tachyonic and positivity of the second derivative tells us that graviton is not a ghost.

It is well-known that $f(R)$ models are equivalent to models in which a scalar field minimally couples to gravity with an appropriate potential function. In fact, we may use a new set of 
variables

$$
\begin{gathered}
\bar{g}_{\mu \nu}=\Omega g_{\mu \nu} \\
\phi=\frac{M_{p}}{2 \beta} \ln \Omega
\end{gathered}
$$

where $\Omega \equiv \frac{d f}{d R}=f^{\prime}(R)$ and $\beta=\sqrt{\frac{1}{6}}$. This is indeed a conformal transformation which transforms the above action in the Jordan frame to the following action in the Einstein frame [11] [15]

$$
S_{E F}=\frac{1}{2} \int d^{4} x \sqrt{-\bar{g}}\left\{\frac{1}{M_{p}^{2}} \bar{R}-\bar{g}^{\mu \nu} \nabla_{\mu} \phi \nabla_{\nu} \phi-2 V(\phi)\right\}+S_{m}\left(\bar{g}_{\mu \nu} e^{2 \beta \phi / M_{p}}, \psi\right)
$$

All indices are raised and lowered by $\bar{g}_{\mu \nu}$. In the Einstein frame, $\phi$ is a minimally coupled scalar field with a self-interacting potential which is given by

$$
V(\phi(R))=\frac{M_{p}^{2}\left(R f^{\prime}(R)-f(R)\right)}{2 f^{\prime 2}(R)}
$$

Note that the conformal transformation induces the coupling of the scalar field $\phi$ with the matter sector. The strength of this coupling $\beta$, is fixed to be $\sqrt{\frac{1}{6}}$ and is the same for all types of matter fields. In the action (7), we take $\bar{g}^{\mu \nu}$ and $\phi$ as two independent field variables and variations of the action yield the corresponding dynamical field equations. Variation with respect to the metric tensor $\bar{g}^{\mu \nu}$, leads to

$$
\bar{G}_{\mu \nu}=M_{p}^{-2}\left(\bar{T}_{\mu \nu}^{\phi}+\bar{T}_{\mu \nu}^{m}\right)
$$

where

$$
\begin{gathered}
\bar{T}_{\mu \nu}^{\phi}=\nabla_{\mu} \phi \nabla_{\nu} \phi-\frac{1}{2} \bar{g}_{\mu \nu} \nabla^{\gamma} \phi \nabla_{\gamma} \phi-V(\phi) \bar{g}_{\mu \nu} \\
\bar{T}_{\mu \nu}^{m}=\frac{-2}{\sqrt{-\bar{g}}} \frac{\delta S_{m}\left(\bar{g}_{\mu \nu}, \psi\right)}{\delta \bar{g}^{\mu \nu}}
\end{gathered}
$$

are stress-tensors of the scalar field and the matter field system. It is important to note that the two stress-tensors $\bar{T}_{\mu \nu}^{m}$ and $\bar{T}_{\mu \nu}^{\phi}$ are not separately conserved. Instead they satisfy the following equations

$$
\bar{\nabla}^{\mu} \bar{T}_{\mu \nu}^{m}=-\bar{\nabla}^{\mu} \bar{T}_{\mu \nu}^{\phi}=\frac{\beta}{M_{p}} \nabla_{\nu} \phi \bar{T}^{m}
$$

We apply the field equations in a homogeneous and isotropic cosmology described by FriedmannRobertson-Walker spacetime

$$
d s^{2}=-d t^{2}+a^{2}(t)\left\{\frac{d r^{2}}{1-k r^{2}}+r^{2}\left(d \theta^{2}+\sin ^{2} \theta d \phi^{2}\right)\right\}
$$

where $a(t)$ is the scale factor and $k$ determines the spatial curvature. The parameter $k$ can take $0,-1,+1$ corresponding to spatially flat, open and curved universes, respectively. We take $\bar{T}_{\mu \nu}^{m}$ and $\bar{T}_{\mu \nu}^{\phi}$ as the stress-tensors of a pressureless perfect fluid with energy density $\bar{\rho}_{m}$, 
and a perfect fluid with energy density $\rho_{\phi}=\frac{1}{2} \dot{\phi}^{2}+V(\phi)$ and pressure $p_{\phi}=\frac{1}{2} \dot{\phi}^{2}-V(\phi)$. In this case, the equations (9) take the form ${ }^{\dagger}$

$$
\begin{gathered}
3 H^{2}+\frac{3 k}{a^{2}}=M_{p}^{-2}\left(\rho_{\phi}+\rho_{m}\right) \\
2 \dot{H}=-M_{p}^{-2}\left[\left(\omega_{\phi}+1\right) \rho_{\phi}+\rho_{m}\right]+\frac{2 k}{a^{2}}
\end{gathered}
$$

where $\omega_{\phi}=\frac{p_{\phi}}{\rho_{\phi}}$ is equation of state parameter of the scalar field $\phi$, and overdot indicates differentiation with respect to cosmic time $t$. We may write the field equations in terms of relative densities defined by $\Omega_{m}=\frac{\rho_{m}}{\rho_{c}}$ and $\Omega_{\phi}=\frac{\rho_{\phi}}{\rho_{c}}$ where $\rho_{c}=3 M_{p}^{2} H^{2}$ is the critical density. These relative densities then satisfy

$$
\Omega_{m}+\Omega_{\phi}-\Omega_{k}=1
$$

where $\Omega_{k}=\frac{k}{a^{2} H^{2}}$. The conservation equations (12) give

$$
\begin{gathered}
\dot{\rho}_{m}+3 H \rho_{m}=Q \\
\dot{\rho}_{\phi}+3 H\left(\omega_{\phi}+1\right) \rho_{\phi}=-Q
\end{gathered}
$$

where

$$
Q=\frac{\beta}{M_{p}} \dot{\phi} \rho_{m}
$$

is the interaction term. This term vanishes only for $\phi=$ const., which due to (6) it happens when $f(R)$ linearly depends on $R$. The direction of energy transfer depends on the sign of $Q$ or $\dot{\phi}$. For $\dot{\phi}>0$, the energy transfer is from dark energy to dark matter and for $\dot{\phi}<0$ the reverse is true.

Let us consider time evolution of the ratio $r \equiv \rho_{m} / \rho_{\phi}$,

$$
\dot{r}=\frac{\dot{\rho}_{m}}{\rho_{\phi}}-r \frac{\dot{\rho}_{\phi}}{\rho_{\phi}}
$$

If we combine the latter with the balance equations (17) and (18), we obtain

$$
\dot{r}=3 H r\left[\omega_{\phi}+\left(1+\frac{1}{r}\right) \frac{\Gamma}{3 H}\right]
$$

where

$$
\Gamma=\frac{Q}{\rho_{\phi}}=\frac{\beta}{M_{p}} r \dot{\phi}
$$

is the decay rate. Now we apply the holographic relation to dark energy density $\rho_{\phi}$ with $L=H^{-1}$,

$$
\rho_{\phi}=3 c^{2} M_{p}^{2} H^{2}
$$

This gives

$$
\dot{\rho}_{\phi}=6 c^{2} M_{p}^{2} H \dot{H}
$$

\footnotetext{
${ }^{\dagger}$ Hereafter we will use unbarred characters in the Einstein frame.
} 
We combine (23) with (15) to obtain

$$
\dot{H}=-\frac{3}{2} H^{2}\left(1+\frac{\omega_{\phi}}{r+1}\right)(r+1) c^{2}+\frac{k}{a^{2}}
$$

One can easily check that

$$
(r+1) c^{2}=\Omega_{k}+1
$$

which reduces $(25)$ to

$$
\dot{H}=-\frac{3}{2} H^{2}\left(1+\frac{\omega_{\phi}}{r+1}\right)\left(\Omega_{k}+1\right)+\frac{k}{a^{2}}
$$

Substituting this into (24) gives

$$
\dot{\rho}_{\phi}=-9 c^{2} M_{p}^{2} H^{3}\left(1+\frac{\omega_{\phi}}{r+1}\right)\left(\Omega_{k}+1\right)+6 c^{2} M_{p}^{2} H \frac{k}{a^{2}}
$$

When we put the latter together with the holographic relation (23) into the balance equation (18), we obtain

$$
\omega_{\phi}=\frac{(r+1)}{3\left(\Omega_{k}-r\right)}\left(\frac{\Gamma}{H}-\Omega_{k}\right)
$$

Note that there is no non-interacting limit in our case since $\Gamma=0$ corresponds to $\phi=$ const., or equivalently, $\Lambda \mathrm{CDM}$ model. We may use (29) in the relation (21) to obtain

$$
\dot{r}=H(r+1)\left(\frac{\Gamma}{H}-r\right) \frac{\Omega_{k}}{\Omega_{k}-r}
$$

For a further step, we introduce the deceleration parameter which is given by

$$
q=-1-\frac{\dot{H}}{H^{2}}
$$

This together with (27) and (29) results in

$$
q=\frac{1}{2}\left(\Omega_{k}+1\right)\left(\frac{\frac{\Gamma}{H}-r}{\Omega_{k}-r}\right)
$$

From the above expressions, we note that the important quantities $\omega_{\phi}, \dot{r}$ and $q$ which describe evolution of the universe appear as functions of $\Gamma, r$ and $\Omega_{k}$. We will consider two different cases :

1) In a spatially flat universe, $\Omega_{k}=0$ and the relation (30) results in $\dot{r}=0$ or $r=$ constant. It is important to note that this result is independent of the details of the decay rate $\Gamma$ or the interaction $Q$. Since the interaction is given by the shape of the $f(R)$ function we conclude that applying holographic principle to the dark energy density $\rho_{\phi}=3 c^{2} M_{p}^{2} H^{2}$ in a spatially flat universe necessarily leads to a constant ratio of energy densities $r=\rho_{m} / \rho_{\phi}$, irrespective of the form of the $f(R)$ function. The reasoning is simple : from the holographic relation (23) one infers that $\rho_{\phi}$ scales like the critical density $\rho_{c}=3 M_{p}^{2} H^{2}$. As a consequence, the relative density corresponding to $\phi$ must be a constant so that $\Omega_{\phi}=\frac{\rho_{\phi}}{\rho_{c}}=c^{2}$. With this result and the fact that in a spatially flat universe $\Omega_{k}=0$, the Friedmann equation (16) results in 
$\Omega_{m}=1-c^{2}$. Thus $\rho_{m}$ has the same scaling as $\rho_{\phi}$ and the ratio $r$ is a constant. In the flat case, the relations (29) and (32) reduce to

$$
\begin{gathered}
\omega_{\phi}=-\left(1+\frac{1}{r}\right) \frac{\Gamma}{3 H} \\
q=\frac{1}{2 r}\left(r-\frac{\Gamma}{H}\right)
\end{gathered}
$$

Contrary to the constancy of $r$, accelerating expansion requires particular configurations of the $f(R)$ function. This is clear from the expression (34) which the requirement that $q<0$ automatically sets a constraint on the decay rate.

The relation (34) indicates a signature flip of the deceleration parameter during expansion of the universe and evolution of $\frac{\Gamma}{H}$. When $\frac{\Gamma}{H}<r$, we have $q>0$ and the expansion is decelerating. The condition $\frac{\Gamma}{H}=r$ defines a transition region in which $q=0$ and the universe enters the accelerating phase for which $\frac{\Gamma}{H}>r$. It should be noted that the deceleration parameter corresponding to a viable $f(R)$ gravity model should exhibit this signature flip in a special way so that

$$
\frac{d q(z)}{d z}>0 \quad \text { for } \quad \frac{\Gamma}{H}=r
$$

It means that we should have an accelerating phase after the decelerating one in the expansion history of the universe. Moreover, the transition region $\frac{\Gamma}{H}=r$ should lie in recent past. These conditions put some constraints on the shape of $f(R)$ functions. This situation is very similar to the case presented in [10] when the Hubble scale is taken as the cutoff length. In that case accelerated expansion constrains the shape of the interaction term of an interacting holographic dark energy model.

2) Although a flat universe is usually assumed, it is still quite possible that there is a contribution to the field equations from the spatial curvature [16]. In a spatially curved universe, the relation (32) indicates that $\frac{\Gamma}{H}=r$ remains as the point in which $q$ changes its sign.

If we combine (30) and (32) and then use the relation (16), we arrive at

$$
\dot{r}=2 q(r+1) H \frac{\Omega_{k}}{\Omega_{k}+1}=2 q H \frac{\Omega_{k}}{\Omega_{\phi}}
$$

which relates evolution of $r$ to the deceleration parameter $q$ and $\Omega_{k}$. This implies that $\dot{r}$ changes its sign at the same point that $q$ does, namely at $\frac{\Gamma}{H}=r$. As the relations (30) and (36) imply, contrary to the spatially flat case, $r$ is not a constant and evolves during expansion of the universe. Although the density parameter $\Omega_{\phi}$ is still a constant equal to $c^{2}$, due to the non-vanishing spatial curvature the Friedmann equation (16) generally gives different scalings for $\rho_{m}$ and $\rho_{\phi}$. The rate of change of the ratio $r$ is closely related to the sign of $q$. In a spatially closed universe $\left(\Omega_{k}>0\right)$ the ratio $r$ is an increasing function of time when the expansion is decelerating $(q>0)$ and it is a decreasing function when the expansion is accelerating $(q<0)$. In a spatially open universe $\left(\Omega_{k}<0\right)$ the reverse is true.

There are two important contributions to the changes of $r$, namely, from $\Omega_{k}$ and $q$. There are some indications [16] that contributions from the spatial curvature should be very small $\left(\left|\Omega_{k}\right| \sim 0.001\right)$. On the other hand, changes of $r$ do not also receive strong contributions from 
$q$ by noting the fact that accelerating expansion of the universe is a recent phenomenon. To clarify this point we first note that transition to the accelerating phase takes place when $\frac{\Gamma}{H}=r$. Assuming that this condition is satisfied in an appropriately small redshift near the present epoch we conclude that we live in an epoch in which $\frac{\Gamma}{H} \approx r$. The relation (30) then indicates that $\dot{r}$ receives a small contribution from the deceleration parameter. This is consistent with the alleviation of the coincidence problem since besides the possibility that the present epoch may be a stationary regime at which the ratio $r$ is a constant, it is also quite possible that we live in a very special epoch, a transient epoch at which the ratio varies slowly with respect to the expansion of the universe ${ }^{\ddagger}$.

In summary, applying holographic principle to $f(R)$ models in a spatially flat universe necessarily leads to a stationary energy density ratio independent of the form of the $f(R)$ function. In a spatially curved universe, although $r$ does not appear as a constant ratio and details of its evolution is model-dependent, all cosmologically viable $f(R)$ models for which the transition region $\frac{\Gamma}{H}=r$ lies in recent past lead to $r \approx$ constant and can alleviate the coincidence problem.

\section{References}

[1] S. Weinberg, Rev. Mod. Phys. 61, 1 (1989)

[2] A. G. Riess, Astrophys. J. 560, 49, (2001)

[3] Y. Bisabr and H. Salehi, Class. Quantum Grav. 19, 2369 (2002)

Y. Bisabr, Gen. Relativ. Gravit. 42, 1211 (2010)

[4] S. M. Carroll, A. De Felice, V. Duvvuri, D. A. Easson, M. Trodden and M. S. Turner, Phys. Rev. D 71, 063513, (2005)

G. Allemandi, A. Browiec and M. Francaviglia, Phys. Rev. D 70, 103503 (2004)

X. Meng and P. Wang, Class. Quantum Grav. 21, 951 (2004)

M. E. soussa and R. P. Woodard, Gen. Rel. Grav. 36, 855 (2004)

S. Nojiri and S. D. Odintsov, Phys. Rev. D 68, 123512, (2003)

[5] G. 't Hooft, gr-qc/9310026

L. Susskind, J. Math. Phys. 36, 6377, (1995)

[6] J. D. Bekenstein, Phys. Rev. D 7, 2333, (1973)

J. D. Bekenstein, Phys. Rev. D 9, 3292, (1974)

\footnotetext{
$\ddagger$ The relation $(36)$ is equivalent to $\dot{r}=\frac{2 q \Omega_{k}}{c^{2}} H$. Following the above discussion we may write $\frac{2 q \Omega_{k}}{c^{2}}<<1$ and then $\dot{r}<<H$, the rate of change of $r$ is slower than expansion of the universe.
} 
J. D. Bekenstein, Phys. Rev. D 23, 287, (1981)

J. D. Bekenstein, Phys. Rev. D 49, 1912, (1994)

[7] A. G. Cohen, D. B. Kaplan and A. E. Nelson, Phys. Rev. Letts. 82, 4971, (1999)

[8] H. D. S. Hsu, Phys. Letts. B 594, 13, (2004)

M. Li, Phys. Letts. B 603, 1, (2004)

[9] D. Pavon and W. Zimdahl, Phys. Letts. B 628, 206 (2005)

D. Pavon and W. Zimdahl, Class. Quantum Grav. 24, 5461 (2007)

[10] S. del Campo, J. o. C. Fabris, R. Herrera and W. Zimdahl, Phys. Rev. D 83, 123006 (2011)

[11] G. Magnano and L. M. Sokolowski, Phys. Rev. D 50, 5039 (1994)

[12] Y. M. Cho, Class. Quantum Grav. 14, 2963 (1997)

E. Elizalde, S. Nojiri and S. D. Odintsov, Phys. Rev. D 70, 043539 (2004)

S. Nojiri and S. D. Odintsov, Phys. Rev. D 74, 086005 (2006)

S. Capozziello, S. Nojiri, S. D. Odintsov and A. Troisi, Phys. Lett. B 639, 135 (2006)

[13] Y. Bisabr, Phys. Rev. D 82, 124041 (2010)

[14] A. D. Dolgov, and M. Kawasaki, Phys. Lett. B 573,1 (2003)

[15] K. Maeda, Phys. Rev. D 39, 3159 (1989)

D. Wands, Class. Quant. Grav. 11, 269 (1994)

[16] M. Tegmark et al, Phys. Rev. D 69, 103501 (2004)

K. Ichikawa, M. Kawasaki, T. Sekiguchi and T. Takahashi, JCAP 0612, 005 (2006)

U. Seljak, A. Slosar and P. Mc Donald, JCAP 0610, 014 (2006)

Y. Wang and P. Mukherjee, Phys. Rev. D 76, 103533 (2007) 